\title{
PROPUESTA PARA EL ANALISIS DE LA POLITICA FISCAL
}

\section{Proposal of scrutiny for the taxing policy}

\author{
Gloria Estela Cárdenas Gómez \\ José G. Vargas-hernández ${ }^{2}$
}

Resumen: El trabajo que se presenta, tiene por objetivo analizar la política fiscal en México. Este análisis pretende ser el fundamento teóricoconceptual que como estructura medular sirva de base para la investigación sobre política fiscal en México. Aunque la situación puede parecer muy compleja, la investigación parte del supuesto que la aplicación de la actual políticas fiscal federal tiene implicaciones en el desarrollo económico en general. Se incluye los estudios empíricos sobre el tema a tratar, así como el modelo de política fiscal.

Palabras clave: Crecimiento económico.Desarrollo regional desigual.Federalismo fiscal. Política fiscal y reforma fiscal integral.

\begin{abstract}
The work presented here, is to analyze fiscal policy in Mexico. This analysis aims to be the theoreticaland conceptual foundation as acor estructure as a basis forrese archon fiscal policy in Mexico. Although the situation may seem very complex, rese archon the assumption that the application of the current federal tax policy ha simplications for economic development ingeneral. Empirical studies on the topic, as well as themodel of fiscal policy are included.
\end{abstract}

Keywords: Economicgrowth. Uuneven regionaldevelopment. Fiscal federalism. Fiscal policyandcomprehensivetaxreform.

1 Profesor Titular C de Tiempo Completo Instituto Tecnológico de Cd. Guzmán. E-mail:jvargas2006@gmail.com

2 M.B.A.; Ph.D. Centro Universitario de Ciencias Económico Administrativas, Universidad de Guadalajara. E-mail: jvargas2006@gmail.com 


\section{INTRODUCCIÓN}

Una de las funciones de la política fiscal es el uso del sistema tributario y el gasto público para influir en la economía contribuyendo a suavizar los ciclos económicos en pos de la estabilidad macroeconómica, por lo que se justifica ampliamente el estudio de la política fiscal. El papel de la política fiscal ha recobrado importancia a raíz de la crisis internacional en el 2008. Cuestas Caza (2013), comenta que la política fiscal es un elemento que fomenta la cohesión social mediante la capacidad para recabar ingresos financieros que destina a financiar el gasto público y lo redistribuye en un marco de justicia social.

La influencia de la política fiscal fue también señalada por la OCDE (2008), destacando que:

Las decisiones en materia fiscal no se toman en el vacío y están sujetas a restricciones determinadas políticamente. La política fiscal es importante porque la fiscalidad esta inextricablemente unida a la naturaleza del estado de bienestar, cuya configuración es un proceso fundamentalmente político. En definitiva, la economía política importa y, por ello, será necesario incluir la consideración de las restricciones políticas en el diseño técnico de los sistemas fiscales, con miras a sembrar expectativas de reforma duraderas en la región.

El marco conceptual para analizar la política fiscal en México se puede enmarcar dentro del estudio de la política económica, que a la vez lleva al estudio de las finanzas públicas y política presupuestaria enmarcada dentro de las políticas públicas. Por lo tanto, en este apartado se analizan varios conceptos que ayudaran a entender lo que es la política fiscal.

\section{CONCEPTO DE POLÍTICA FISCAL}

A continuación se analizará el concepto de política fiscal, el cual debe quedar claro para poder avanzar en el análisis que tiene por objeto este trabajo de investigación. La idea es 
confrontar varios conceptos y de estos sacar uno propio para poder manejarlo a lo largo de la investigación.

El Diccionario de Economía Aplicada de Galindo Martin (coord.) (2008: 383), define a la política fiscal como el "conjunto de variaciones en los programas de ingreso y gastos públicos que lleva a cabo el decisor político, con el objeto de alcanzar los diferentes objetivos de política económica, tales como un cierto nivel de empleo, una tasa tolerable de inflación, crecimiento económico, etc.” Por otra parte, el Diccionario Enciclopédico de Economía, Finanzas y Empresa de Merino Peral y López García (2006: 371) define a la política fiscal de la siguiente manera: "Junto con la monetaria, forman la base de actuación de la política económica de un gobierno para conseguir la estabilidad y los objetivos de política macroeconómica. Puede ser también un instrumento eficaz para lograr la redistribución de la renta y de la riqueza”.

El Diccionario de Ciencias Económicas Administrativas de Cárdenas Cutiño (2008: 589), define a la política fiscalcomoel recurso con el que el gobierno influye en la economía por medio de su presupuesto al cambiar los impuestos y pagos de previsión y/o gasto gubernamental. Consiste en el proceso de orientación de la actividad económica a través del instrumento tributario, para conseguir determinados objetivos. Se ocupa de los efectos de los impuestos, gastos y deuda pública sobre los niveles de ingreso nacional, así como de la posibilidad de usar los programas fiscales para producir efectos socialmente deseables sobre la economía. Los objetivos que persigue la política fiscal pueden ser: fomentar el ahorro interno y el comercio exterior, desalentar el contrabando, aumentar el bienestar social, modificar la distribución del ingreso, evitar el desempleo, etcétera.

Por otra parte, Erías y Sánchez Santos (2008), definen la política fiscal "como el conjunto de decisiones que afectan el nivel o la composición del gasto público, así como el peso, estructura o formación de los pagos impositivos". Para Taylor y Weerapana(2011: 509), simplemente: "La política guberna- 
mental que tiene que ver con los impuestos, gastos y el endeudamiento se llama política fiscal". Para Córdoba Padilla (2009: 10), la política fiscal dirige y controla gastos e ingresos, así como la administración de la deuda publica en una forma que tiene pleno conocimiento del efecto de estas operaciones sobre la asignación de los recursos y la corriente de fondos y, por tanto, de su influencia sobre los niveles de renta, precios empleo y producción.

Para el Centro de Emprendedores de la Administración Pública (s.f.), la Política fiscal es "el conjunto de instrumentos y medidas que toma el estado con el objeto de recaudar los ingresos necesarios para realizar las funciones que le ayuden a cumplir los objetivos de la política económica general". Para Samuelson y Nordhaus (s.f.), la política fiscal:Es una rama de la política económica que configura el presupuesto del Estado, y sus componentes, el gasto público y los impuestos, como variables de control para asegurar y mantener la estabilidad económica, amortiguando las variaciones de los ciclos económicos, y contribuyendo a mantener una economía creciente, de pleno empleo y sin inflación alta.

De acuerdo a lo anterior se puede comprobar que no existe una definición exacta de política fiscal, pero al confrontar varias definiciones dan una idea más concreta que ayuda a elaborar un concepto propio que se maneja en este trabajo, el cual se presenta a continuación:

Se considera a la política fiscal, como una herramienta de estabilización macroeconómica, de redistribución del ingreso y de riqueza, utilizada por el Estado(federal, estatal o local),cuyos componentes principales son el gasto público, los impuestos y la deuda pública, llegando a materializarse por medio del presupuesto del Estado. Primeramente se considera una herramienta de estabilización macroeconómica de acuerdo a la teoría keynesiana, por medio de la cual el Estado interviene en la economía, tratando de disminuir el impacto de los ciclos económicos, aumentando la demanda por medio del 
gasto público y la inversión. En segundo lugar es considerada también una herramienta por medio de la cual el Estado puede llevar a cabo la redistribución del ingreso y riqueza como parte de las funciones básicas que este tiene.

Cabe aclarar que la palabra "fiscal" se relaciona con el fisco o con la hacienda pública y no debe confundirse con tributación (tributar) o impuestos, ya que estos no son los únicos ingresos que obtiene el fisco. La política fiscal incluye el financiamiento o deuda del sector público. Para algunos la terminología adecuada debería ser ciencia fiscal (la negrita es nuestra) por "todas las complicaciones que de este accionar se determinen, incluyendo las fuerzas socioeconómicas que las provocan" (RetchkimanK., 1972: 82).Los componentes principales de la política fiscal que son el gasto público, los impuestos y la deuda pública.

\section{MARCO TEÓRICO DE LA POLÍTICA FISCAL}

El análisis integrado de la economía y la política tiene un tratamiento desde los tiempos de inicio del liberalismo (o incluso mucho tiempo atrás) con Adam Smith y reafirmado con el pensamiento Keynesiano, así como a los que le han seguido, y es que en realidad no se puede separar estas dos ciencias, ni tampoco debemos darle un tratamiento separado, ya que los efectos de una impactan a la otra y viceversa.

En este apartado se aborda de manera general las principales teorías relacionadas con el tema a tratar sobre política fiscal, haciendo énfasis en las teorías sobre política fiscal del lado de la oferta y de lado de la demanda, así como las teorías sobre el Estado y teoría de los impuestos. Se hará referencia a la bibliografía disponible, de tal manera que sea posible para el lector recurrir a estas fuentes para profundizar más en los contenidos propuestos. Por ser estas teorías generales, a excepción de algunos detalles de presentación, no hay que se me pueda atribuir de original y personal. 


\section{A política fiscal del lado de la oferta}

\section{Teoría clásica o liberalismo económico clásico}

El liberalismo económico se considera una doctrina o pensamiento económico que pone un excesivo énfasis en el laissez-faire(dejar ser, dejar pasar), destacando la libertad de actuación de la iniciativa privada y en donde son las fuerzas del mercado son las que establecen los precios y los salarios, situación que se presenta dentro del sistema capitalista imperante. Por lo tanto, el liberalismo económico fundamenta la libertad e iniciativa individual, la propiedad y la empresa privadas con una interferencia mínima del gobierno (BrueyGrant, 2009).Los siguientes elementos resultan de la premisa liberal básica que juntos constituyen la ideología liberal, los cuales son presentados por Shively (1997):

1) La democracia de algún tipo es la forma apropiada de gobierno

2) Las personas deben tener libertad intelectual completa, incluyendo libertad de expresión, religiosa y de prensa. (Deben tener la responsabilidad de sus propios valores, por lo que deberían desarrollar la capacidad de juzgar valores.)

3) El poder gubernamental debe ser mínimo y deberá regular muy poco las vidas de las personas. (Deberá tomarse la menor cantidad de decisiones posible por las personas, de modo que ellas aprendan a tomar decisiones por sí mismas.)

4) En particular, las personas deberán ser libres de regular su propia actividad económica (Como lo anterior).

5) El poder de una persona sobre otra es algo malo: por tanto el gobierno deberá organizarse para resguardar contra los abusos de poder (una vez más, una persona no debería tomar decisiones por otra). 
El liberalismo económico impugnaba por limitar la intervención del Estado en la vida económica, ya que anteriormente durante el mercantilismo que opero desde el Siglo XVI, el Estado tuvo una participación muy amplia ejercida por un control total de la economía y al tener el poder político y económico, le permitió realizar grandes conquistas llevando a cabo el proceso de colonización, imponiendo impuestos a los pueblos conquistados y creando grandes monopolios sobre algunos productos como la sal y la acuñación de moneda, así como el control absoluto del comercio interno y externo. De esta forma el liberalismo trataba de sacudirse el yugo que representaba el Estado, de allí el nombre, que programaba la libertad en toda la extensión de la palabra: individual, de expresión, de ejercer el comercio, etc.

Para el liberalismo el Estado debería reducir al mínimo su participación en la economía, asignándole funciones básicas como asegurar el mantenimiento del orden y la vigilancia del cumplimiento de la ley, como garantías para el buen funcionamiento de la sociedad. El liberalismo económico fue introducido no por los líderes comerciales e industriales, sino por los intelectuales del siglo XVII y XVIII, quienes alentados por la intranquilidad artística y científica general de la época crearon la teoría clásica.

Para mencionar algunos de los propulsores del liberalismo económico encontramos a filósofos como Thomas Hobbes (1588-1679) y la influencia de Jean Bodis (1530-1596) y Francis Bacon (1561-1626); Bernard de Mandeville (1670-1733); "La escuela del sentido moral": Antony Ashley Cooper Conde de Shaftesbury (1671-1713), Joseph Butler (1692-1752), Francis Hutcheson (1694-1746). De la escuela clásica francesa encontramos al Dr. FrancoisQuesnay (1694-1774), Anne-Robert-Jacques Turgot, Baron de L'Aune (1727-1781) y a EtianneBonnot, abate de Condillac (1715-1780). De la escuela clásica Inglesa tenemos al célebre Adam Smith (1723-1790), a Thomas Robert Malthus (1766-1834) y a David Ricardo (1772-1823, así 
como los continuadores de la escuela clásica inglesa como John Stuart Mill (1773-1836), Jean BaptisteSay (1760-1830).

\section{a) Los economistas clásicos}

Sus estudios que datan de mediados del siglo XVIII y mediados del XIX y se centran en el análisis social y político sobre todo en el crecimiento económico. Llevaron las leyes de la física, enunciado por Newton, al ámbito económico creando las bases de microeconomía y macroeconomía ciencias que se estudian hoy en día en la mayoría de las universidades. Los clásicos creían que las leyes de la economía son universales e inmutables (BrueyGrant, 2009: 47).

Respecto a las fallas de la teoría clásica, Brue y Grant(2009: 49) comenta que además de su excesivo énfasis en el laissez-faire, la economía clásica era ambigua, deficiente o errónea en varias áreas del análisis económico. La historia y los teóricos económicos subsiguientes mostrarían que el laissez-faire, como una política pública era inadecuada para enfrentarse, entre otras cosas a las depresiones económicas, los monopolios (ya fueran naturales o no), al poder monopsonio, a los efectos externos de las acciones privadas y a las provisiones de bienes cuyos beneficios eran indivisibles (bienes públicos).

\section{2) La teoría de la riqueza de las naciones y la mano invisible de Adam Smith}

Adam Smith (1723-1790) considerado como el teórico económico más importante del siglo XVIII, es llamado el padre del liberalismo económico. Su obra "El origen de la riqueza de las naciones" es la obra más relevante del liberalismo económico. Su teoría principal se sustenta en el argumento de las decisiones económicas individuales son guiada por "una mano invisible" para incrementar el bienestar de la sociedad. Las obras públicas, la educación pública y la promoción de la cultura son 
actividades del Estado por ser poco rentables. El comercio internacional debe fundamentarse en el intercambio de la ventaja absoluta que tienen las naciones en sus factores de producción.

\section{a) Seguidores de Adam Smith}

David Ricardo quien con su obra"Principios de economía política y tributación" (1817) da sustento a la teoría del valor, teoría de la renta de la tierra y al comercio internacional, mediante la ventaja comparativa. Realiza aportaciones a las leyes de los rendimientos marginales decrecientes, los salarios y los precios de los productos. Por otra parte, Thomas Malthus, en su "Ensayo sobre el principio de la población" (1798) argumenta que el crecimiento de la población es un obstáculo para el crecimiento económico por lo que esta debería ser controlada.

\section{A política fiscal del lado de la demanda}

La política fiscal del lado de la demanda nos lleva a analizar la teoría de John Maynard Keynes la cual se presenta a continuación:

\section{1) Teoría general del empleo, el interés, y el dinero}

En 1936, John Maynard Keynespublicó su obra "Teoría general del empleo, el interés, y el dinero" que también se conoce como la Teoría Keynesiana, o como el modelo del Estado Benefactor que analiza las variaciones de la demanda agregaday sus relaciones con el empleo y de ingresos. Frente a la crisis financiera conocida como la Gran depresión, los gobiernos y el sector privado parecían impotentes para resolverla. Keynes considera que la situación normal del capitalismo del laissez faire es un nivel de empleo fluctuante, contrario a la teoría clásica que afirma que siempre hay una tendencia hacia el empleo total. La gran tarea que se impuso a si mismo fue explicar la persistencia del desempleo (Posner, 2010: 260). Su propuesta se centra la 
aplicación de impuestos progresivos, la regulación de las inversiones y la generación del empleo.

\section{1) Déficit presupuestario y Teoría del Multiplicador}

La teoría del multiplicador es un concepto aplicable a la relación inversión-ingreso relacionada con el consumo a largo plazo. El incremento en el gasto público en tiempos de depresión económica promueve la recuperación de los salarios y la recuperación de las empresas, lo cual mediante el mecanismo del multiplicador, incrementa la recaudación de impuestos. Esta recomendación sobre el déficit presupuestario se toma con reservas porque el incremento de la deuda pública compromete a las generaciones futuras. Esta es una de las razones por las cuales la teoría keynesiana y sobretodo la neo-keynesiana, es criticada.

El aporte Keynesiano revaloriza el papel de la política fiscal en coordinación con la política monetaria para consolidar las oportunidades que mediante la intervención del gobierno se crean para el estableciendo de una economía de pleno empleo. Las medidas de política fiscal relacionan los gastos públicos con los impuestos (Asorey, s.f.).

\section{A otras teorías relacionadas con la política fiscal}

Se presentan a manera de resumen las tres principales teorías que se relacionan o tienen que ver con la política fiscal, las cuales se presentan a continuación:

\section{1) Teoría de la economía mixta}

Con el cambio de paradigma que trajo la Revolución Keynesiana y bajo el escenario de la gran depresión de los años treinta, se puso en práctica en varios países un nuevo sistema llamado economía mixta con el propósito de superar la crisis mundial que se presentó en aquella época. Por economía mixta 
se entiende el sistema económico en donde la propiedad de los medios de producción de propiedad privada o del Estado interactúan entre las empresas y el sector público para la producción, distribución y consumo de bienes y servicios, bajo la intervención, regulación y planificación del Estado. Esta política se implementa en 1933 con el New Deal de Roosevelt coincidiendo con el Keynesianismo.La economía mixta se caracteriza por una fuerte intervención del Estado en la economía a través de la política económica orientada a la creación de las empresas estatales, control de precios, y control del mercado.

\section{a) Bases constitucionales que evidencian la aplicación de la economía mixta en México}

Los artículos 5, 25 y 28 de la Constitución Política de los Estados Unidos Mexicanos (CPEUM, 2015) establecen el ordenamiento de la economía de sistema mixto en México.

\section{2) Teoría del estado de bienestar}

El Estado de Bienestar surge como un concepto que sustituye a la riqueza por el de bienestar que incorpora juicios normativos a la atención de las necesidades sociales, dando por resultado una política social que asume la responsabilidad de proveer el bienestar a la sociedad en términos de salud, educación, vivienda, alimentación, empleo, pensiones, seguro de desempleo, etc.

\section{3) Teoría del estado}

La teoría del estado proporciona un marco teórico a la formulación e implementación de la política fiscal. El Estado se define como una población que forma una comunidadpolítica asentada en un territorio y organizada con un gobierno soberano. 


\section{4) La teoría de la "estatidad"}

La teoría de la "estatidad" argumenta la existencia de un proceso constitutivo en la formación del Estado que se define en los diferentes componentes que estructuran la organización social hasta adquirir los atributos esenciales entre otros la externalización del poder, la institucionalización de la autoridad, procesos de diferenciación de control e internalización de una identidad colectiva (Oszlak,1982, Jan. - Mar.).

\section{5) Teoría de los impuestos}

Esta teoría se enfoca al estudio de los impuestos, sus impactos y efectos en las decisiones económicas en los contribuyentes, ciudadanos, trabajadores, empresas y en general a la población en variables tales como la producción, consumo, precios, ahorro, inversión, crédito, tasas de intereses, comercio y finanzas internacionales, etc. Uno de los primeros estudios sobre los principios fundamentales y características esenciales de los tributos es el realizado por Adam Smith en su Libro V de su "Investigación de la Naturaleza y Causas de la Riqueza de las Naciones".Los principios siguen siendo fundamentales para el estudio de los impuestos enunciados en términos de justicia o proporcionalidad,certidumbre o certeza, comodidad y economía.

El principio de justicia considera que todos sin excepción tienen la obligación de contribuir en forma igualitaria con el pago de sus impuestos en proporción a sus capacidades. La observancia de este principio conlleva la equidad horizontal y vertical de los impuestos. La equidad es horizontal cuando las personas en la misma situación económica pagan los mismos impuestos. La equidad es vertical cuando los que más tienen pagan más impuestos(Caballero Urdiales, 2006).

Bajo el principio de certidumbre se desprenden entre otras consideraciones de que las obligaciones de las personas a pagar impuestos fijos, no arbitrarios y que proporcionen la 
certeza, respetada con claridad por los legisladores en todos sus elementos como el sujeto, objeto, exenciones, época y tarifa de pago, sanciones e infracciones para los obligados. Arrioja Vizcaíno (2010) caracteriza el principio de certidumbre como los elementos de las contribuciones se establecen con precisión y certeza en las leyes, con claridad, en lenguaje inteligible y llano de tal forma que los obligados entiendan y no evadan sus obligaciones porque son causa de altanerías y corrupción.

Bajo el principio de la comodidad, se prescribe que la recaudación debe hacerse en la forma y tiempo que convengan al contribuyente y a través de mecanismos que permitan el cumplimiento con facilidad en el momento oportuno, en el lugar adecuado y en forma precisa y económica. Este principio requiere de simplificación de procedimientos y trámites a través de medios electrónicos para la presentación de declaraciones y pagos. El contribuyente debe recibir la atención efectiva y oportuna para que cumpla con comodidad con sus obligaciones fiscales. Arrioja Vizcaíno (2010) tipifica y caracteriza el principio de la comodidad en la elección de términos, períodos y épocas, lugares y trato, mecanismos facilitadores, procedimientos y documentación que resulten cómodos, ventajosos y propicios para quienes tienen la obligación tributaria.

El principio de economía se refiere a la optimización y eficiencia en la administración de los recursos. En los procesos de recaudación se deben considerar la relación costo - beneficio. Es decir, el gasto que se haga para recaudar los ingresos no deba ser superior a lo que se obtenga a través de la recaudación: evaluar el costo-beneficio de la recaudación (principio que no ha sido fácil cumplir por parte de la autoridad).Respecto de este principio, Adam Smith (1936), señala cuales son las cuatro causas por las que se califica de antieconómico un impuesto: 1) El empleo de un gran número de funcionarios para el cobro de los impuestos (inflar la nómina significa aumento de gastos), 2) Los impuestos opresivos a la industria (desanima al inversionista e impide de esta forma la creación de empleos), 3) Las 
confiscaciones y penalidades (que en ocasiones provocan el cierre de fuentes de empleo e ingresos futuros para el Estado), 4) Las visitas y fiscalización por parte de los recaudadores (llegando en ocasiones a ser medios para llevar acabo abusos por parte de la autoridad fiscalizadora).

\section{A tipos de política fiscal}

La política fiscal puede ser de dos tipos: 1) Política fiscal expansiva y 2) Política fiscal contractiva.

La forma de combinación de los elementos de ingresos y gastos dependerá de la situación económica que se atraviese en ese momento, aunque también influye fuertemente de manera directa "la ideología del diseñador y ejecutor" de la política fiscal. En el caso de que exista baja actividad económica se recomienda implementar una política fiscal expansiva que consiste en aumentar los gastos públicos combinado con una disminución de impuestos.

En el caso contrario cuando la economía se encuentra en pleno apogeo, con un crecimiento económico sostenido, se recomienda implementar una política fiscal contractiva que consiste en aumentos de los impuestos y recortes en el gasto público; de esta forma se evita o disminuye el impacto de los siglos económicos y el paro (sinónimo de desocupación) en la economía, por lo que se justifica de esta forma la intervención del Estado.

La combinación que se logra con los elementos del gasto público y los impuestos produce efectos directos en la economía a corto plazo como se mencionan a continuación:

1) Lograr un equilibrio en la economía (sobre todo en tiempo de crisis)

2) Demanda agregada: efecto en la producción, empleo y precios del mercado

3) Actividad económica y/o Crecimiento económico 
4) La retribución de la riqueza

5) Administración adecuada de recursos

6) Nivel de endeudamiento e inversión.

\section{INVESTIGACIONES EMPÍRICAS REALIZADOS SOBRE POLÍTICA FISCAL}

Se ha escrito y publicado mucho sobre política Fiscal y su impacto en el desarrollo regional en México, pero sobre todo, sobresalen los estudios sobre América Latina y el Caribe que tanto la CEPAL como el Banco Mundial han publicado.En este apartado solo se hace referencia a los trabajos de investigación empíricos realizados sobre México que tienen relación sobre el tema a tratar, específicamente sobre el análisis de la política fiscal en México y que apoyan la investigación a realizar. En el cuadro 1,se presentan a manera de resumen, algunos de los diversos trabajos de investigadores relevantes y representativos seleccionados sobre el análisis de la política fiscalen México, del periodo comprendido del 2000 a la fecha.

Cuadro 1: Estudios empíricos sobre el análisis de la política fiscal en México

Periodo: 2000-2014

\begin{tabular}{|l|l|}
\hline Autor & $\begin{array}{l}\text { Estada Alvarado, M. M. y Gómez Camacho, N. } \\
(2000) .\end{array}$ \\
\hline Tema & $\begin{array}{l}\text { La política fiscal en México como palanca de creci- } \\
\text { miento: Estimulo u obstáculo. }\end{array}$ \\
\hline $\begin{array}{l}\text { Conclu- } \\
\text { siones }\end{array}$ & $\begin{array}{l}\text { La política fiscal tiene un papel relevante en el de- } \\
\text { sarrollo de los países. } \\
\text { En México la política fiscal es un instrumento de } \\
\text { política económica y no un regulador del ciclo } \\
\text { económico. }\end{array}$ \\
\hline
\end{tabular}




\begin{tabular}{|c|c|}
\hline Autor & Manzano, B. \& Ruiz, J. (2004). \\
\hline Tema & Política fiscal óptima: El estado de la cuestión. \\
\hline $\begin{array}{l}\text { Conclusio- } \\
\text { nes }\end{array}$ & $\begin{array}{l}\text { La implementación de "la política Iscal óptima" } \\
\text { en diferentes contextos, no son muy dispares o } \\
\text { diferentes considerando una "estructura de pref- } \\
\text { erencias comúnmente utilizada, separable en el } \\
\text { tiempo y homotética". }\end{array}$ \\
\hline Autor & Vázquez López, R. (2006, junio). \\
\hline Tema & $\begin{array}{l}\text { Los antecedentes del estancamiento económico: } \\
\text { la política fiscal en México de 1984-1994 }\end{array}$ \\
\hline $\begin{array}{l}\text { Conclusio- } \\
\text { nes }\end{array}$ & $\begin{array}{l}\text { La crisis de } 1994 \text { fue el resultado del fracaso de } \\
\text { las políticas implementadas en los gobiernos an- } \\
\text { teriores y que permanece actualmente. }\end{array}$ \\
\hline Autor & Vargas Téllez, C. O. (2006). \\
\hline Tema & $\begin{array}{l}\text { Aplicada Incidencia Fiscal y del Gasto sobre la } \\
\text { Renta Familiar: Un Enfoque de Micro datos } \\
\text { para México 1984-2002. }\end{array}$ \\
\hline $\begin{array}{l}\text { Conclusio- } \\
\text { nes }\end{array}$ & $\begin{array}{l}\text { Los bajos ingresos fiscales limitan el presupues- } \\
\text { to en la inversión del Estado en la protección } \\
\text { social. Con esto, se avanza poco en el combate } \\
\text { a la pobreza y la disminución de la desigualdad } \\
\text { mientras que la renta se concentra en el } 35 \% \text { de } \\
\text { las familias más ricas que mejoran su posición } \\
\text { relativa y absoluta. }\end{array}$ \\
\hline Autor & Ramírez Cedillo, E. (2006). \\
\hline Tema & Por una política fiscal contra cíclica. \\
\hline $\begin{array}{l}\text { Conclusio- } \\
\text { nes }\end{array}$ & $\begin{array}{l}\text { Las políticas restrictivas implementadas para } \\
\text { promover la inversión extranjera afecta a los sec- } \\
\text { tores de la producción. El incremento de las ta- } \\
\text { sas de interés y la apreciación del tipo de cambio, } \\
\text { debilitan la productividad y la competitividad, } \\
\text { reducen el empleo y el crecimiento económico }\end{array}$ \\
\hline
\end{tabular}




\begin{tabular}{|c|c|}
\hline Autor & Galindo Martín, M. A. (2007, Marzo-Abril). \\
\hline Tema & $\begin{array}{l}\text { Gobernanza, política fiscal y crecimiento } \\
\text { económico. }\end{array}$ \\
\hline $\begin{array}{l}\text { Conclusio- } \\
\text { nes }\end{array}$ & $\begin{array}{l}\text { La gobernanza tiene un efecto positivo sobre el } \\
\text { crecimiento económico como resultado de po- } \\
\text { tenciar la política fiscal mediante rebajas imposi- } \\
\text { tivas y mejora del gasto público. }\end{array}$ \\
\hline Autor & Ramírez Cedillo, E. (2009). \\
\hline Tema & $\begin{array}{l}\text { El abandono de la política fiscal como herra- } \\
\text { mientas de crecimiento. }\end{array}$ \\
\hline $\begin{array}{l}\text { Conclusio- } \\
\text { nes }\end{array}$ & $\begin{array}{l}\text { La ejecución del déficit presupuestal del gobi- } \\
\text { erno tiene efectos multiplicadores internos. La } \\
\text { distribución equitativa del gasto en relación al } \\
\text { ingreso contribuye a un aumento en la demanda } \\
\text { agregada. }\end{array}$ \\
\hline Autor & $\begin{array}{l}\text { Chávez Martín del Campo, J. C., Rodríguez Var- } \\
\text { gas, R. y Fonseca Hernández, F. d.J. (2010, Julio- } \\
\text { Diciembre 25). }\end{array}$ \\
\hline Tema & $\begin{array}{l}\text { Vacas gordas y vacas flacas: La política fiscal y el } \\
\text { balance estructural en México, 1990-2009. }\end{array}$ \\
\hline $\begin{array}{l}\text { Conclusio- } \\
\text { nes }\end{array}$ & $\begin{array}{l}\text { El comportamiento pro cíclico de la política fis- } \\
\text { cal mexicana se relaciona con el balance público } \\
\text { contra cíclico. Esto se revierte en períodos ex- } \\
\text { pansivos cuando aumentan los ingresos y por } \\
\text { tanto, el gasto público. No se tienen los instru- } \\
\text { mentos adecuados para enfrentar los períodos } \\
\text { de bajo crecimiento económico en materia de } \\
\text { gasto estabilizador que atenúen los efectos rece- } \\
\text { sivos o que acumule los excedentes en etapas } \\
\text { expansivas. }\end{array}$ \\
\hline
\end{tabular}




\begin{tabular}{|c|c|}
\hline Autor & $\begin{array}{l}\text { Martin-Mayoral, F. y Uribe, C. A. (2010, julio- } \\
\text { septiembre). }\end{array}$ \\
\hline Tema & $\begin{array}{l}\text { Determinantes económicos e institucionales del } \\
\text { esfuerzo fiscal en América Latina. }\end{array}$ \\
\hline $\begin{array}{l}\text { Conclusio- } \\
\text { nes }\end{array}$ & $\begin{array}{l}\text { La aplicación de políticas de recaudación tribu- } \\
\text { taria debe orientarse a incrementar los niveles } \\
\text { de ingresos, a disminuir los niveles de desigual- } \\
\text { dad y la promoción de las inversiones. }\end{array}$ \\
\hline Autor & Cortes, F. (2010). \\
\hline Tema & Desigualdad económica y poder en México. \\
\hline $\begin{array}{l}\text { Conclusio- } \\
\text { nes }\end{array}$ & $\begin{array}{l}\text { La redistribución progresiva del ingreso tiene } \\
\text { efectos directos de distribución sobre la pobreza } \\
\text { y efectos indirectos de ingreso en elcrecimiento } \\
\text { económico. Se generan procesos virtuosos de } \\
\text { mayor crecimiento económico, y menor desigual- } \\
\text { dad y pobreza. }\end{array}$ \\
\hline Autor & Cruz Núñez, I. (2010). \\
\hline Tema & $\begin{array}{l}\text { Política fiscal en México bajo el modelo neo- } \\
\text { liberal (1982-2009): ¿Obstáculo al crecimiento } \\
\text { económico? }\end{array}$ \\
\hline $\begin{array}{l}\text { Conclusio- } \\
\text { nes }\end{array}$ & $\begin{array}{l}\text { La política fiscal contractiva de México se car- } \\
\text { acterizapor el estancamiento de los ingresos pre- } \\
\text { supuestarios. Un menor gasto público no con- } \\
\text { tribuye al crecimiento económico. El proyecto } \\
\text { de desarrollo económico nacional debe articular } \\
\text { el mercado interno, la equidad en la partici- } \\
\text { pación de los agentes económicos para satisfacer } \\
\text { sus necesidades y mejorar su bienestar. }\end{array}$ \\
\hline
\end{tabular}




\begin{tabular}{|l|l|}
\hline Autor & $\begin{array}{l}\text { Martínez, L. D., CaamalCauich, I. y Ávila } \\
\text { Dorantes, J. A. (2011, Septiembre 10). }\end{array}$ \\
\hline Tema & $\begin{array}{l}\text { La política fiscal y el crecimiento económico en } \\
\text { México. }\end{array}$ \\
\hline $\begin{array}{l}\text { Conclusio- } \\
\text { nes }\end{array}$ & $\begin{array}{l}\text { Los impactos de lareforma estructural de la } \\
\text { política fiscal en México debe aumentar la recau- } \\
\text { dación fiscal, los niveles de ingreso, y eficiente el } \\
\text { gasto público. }\end{array}$ \\
\hline
\end{tabular}

\begin{tabular}{|l|l|}
\hline Autor & Angulo López, E. (2011). \\
\hline Tema & $\begin{array}{l}\text { Política fiscal y estrategia como factor de desar- } \\
\text { rollo de la mediana empresa comercial sina- } \\
\text { loense: Un estudio de Caso. }\end{array}$ \\
\hline $\begin{array}{l}\text { Conclusio- } \\
\text { nes }\end{array}$ & $\begin{array}{l}\text { La mediana empresa Sinaloense enfrentan prob- } \\
\text { lemas de inseguridad, falta de créditos, compe- } \\
\text { tencia de consorcios internacionales, falta de } \\
\text { incentivos fiscales, incremento de la carga fiscal, } \\
\text { burocracia por nuevas cargas administrativas, fal- } \\
\text { ta de asesoría fiscal profesional, incertidumbre } \\
\text { al invertir por obstáculos de tipo laboral, fiscal, } \\
\text { administrativo y gubernamental, etc. }\end{array}$ \\
\hline
\end{tabular}

\begin{tabular}{|l|l|}
\hline Autor & Rodríguez Arana, Z. A. (2012). \\
\hline Tema & $\begin{array}{l}\text { Efectos de las políticas fiscal y monetaria en un } \\
\text { modelo donde se determinan simultáneamente } \\
\text { el ciclo y el crecimiento económico. }\end{array}$ \\
\hline $\begin{array}{l}\text { Conclusio- } \\
\text { nes }\end{array}$ & $\begin{array}{l}\text { Los efectos de la política fiscal sobre el crecimien- } \\
\text { to económico dependen el instrumento fiscal. El } \\
\text { incremento en tasas impositivas aumenta la tasa } \\
\text { de ahorro y generan una recesión económica. } \\
\text { El aumento en el gasto del consumo público } \\
\text { reduce la tasa de ahorro y aumenta el creci- } \\
\text { miento económico. A mayor inversión pública } \\
\text { aumentan los efectos positivos en el crecimiento } \\
\text { económico. La política monetaria expansiva au- } \\
\text { menta el crecimiento económico, aunque no es } \\
\text { neutral al nivel del capital y del producto. }\end{array}$ \\
\hline
\end{tabular}




\begin{tabular}{|l|l|}
\hline Autor & $\begin{array}{l}\text { Rodríguez Nava, A. y Venegas Martínez, F. } \\
\text { (2012). }\end{array}$ \\
\hline Tema & $\begin{array}{l}\text { Criterios y medidas de política económica en } \\
\text { respuesta a la actual crisis económica y finan- } \\
\text { ciera }\end{array}$ \\
\hline $\begin{array}{l}\text { Conclu- } \\
\text { siones }\end{array}$ & $\begin{array}{l}\text { La política fiscal emprendida para frenar la } \\
\text { crisishasido contractiva con medidas macro- } \\
\text { económicas restrictivas para lograr la estabili- } \\
\text { dad de las finanzas públicas. }\end{array}$ \\
\hline Autor & Moleres Regalado, E. (2012). \\
\hline Tema & $\begin{array}{l}\text { Política fiscal, tipo de cambio y tasa natural de } \\
\text { crecimiento en México: Evidencia empírica. }\end{array}$ \\
\hline $\begin{array}{l}\text { Conclu- } \\
\text { siones }\end{array}$ & $\begin{array}{l}\text { Las políticas de estabilización económica en } \\
\text { México en los últimos decenios que han tenido } \\
\text { como objetivo reducir la inflación, han dep- } \\
\text { rimido la demanda efectiva y a un crecimiento } \\
\text { económico. }\end{array}$ \\
\hline Autor & Pliego Quintana, M. (2012). \\
\hline Tema & $\begin{array}{l}\text { Política fiscal en situaciones recesivas: 1993- } \\
\text { 2004. }\end{array}$ \\
\hline $\begin{array}{l}\text { Conclu- } \\
\text { siones }\end{array}$ & $\begin{array}{l}\text { La intervención y las políticas de austeri- } \\
\text { dad presupuestal del Estado mexicano en la } \\
\text { economía entre 1993 y 2004impulsaronel } \\
\text { crecimiento en un periodo de crisis y estanca- } \\
\text { miento económico. La canalización de más re- } \\
\text { cursos al sistema financiero inhibió el gasto pú- } \\
\text { blico en las actividades productivas. La banca } \\
\text { ha recibido los beneficios de los ingresos por la } \\
\text { venta del petróleo. }\end{array}$ \\
\hline
\end{tabular}




\begin{tabular}{|l|l|}
\hline Autor & $\begin{array}{l}\text { Inzunza Mejía, P. C. \& Sánchez Díaz, A. J. } \\
\text { (2013). }\end{array}$ \\
\hline Tema & $\begin{array}{l}\text { Disciplina fiscal: límites de la política fiscal para } \\
\text { promover el desarrollo en México. }\end{array}$ \\
\hline $\begin{array}{l}\text { Conclusio- } \\
\text { nes }\end{array}$ & $\begin{array}{l}\text { La disciplina fiscaltiene limitaciones para man- } \\
\text { tener el equilibrio fiscal, eficiencia y estabilidad } \\
\text { macroeconómica con la recurrente contratación } \\
\text { de deuda pública en un escenario de declive } \\
\text { económico y financiero. Los resultados se refle- } \\
\text { jan en el escaso crecimiento y poca generación } \\
\text { de empleo.El equilibrio fiscal no es neutral a la } \\
\text { política fiscal contra cíclica y restrictiva para las } \\
\text { finanzas públicas. }\end{array}$ \\
\hline Autor & \begin{tabular}{l} 
Pliego Quintana, M. (2014, Mayo-Augusto) \\
\hline Tema
\end{tabular} \\
\hline $\begin{array}{l}\text { Análisis coyuntural de la política fiscal en } \\
\text { México 1993-2004. }\end{array}$ \\
\hline Conclusio- \\
nes
\end{tabular}

Fuente: Elaboración propia (2015). 
En resumen, cada uno de los anteriores investigadores realiza exhaustivos análisis sobre política fiscal y sus afectos en la economía mexicana.El método utilizado en la mayoría de los casos fue la investigación cualitativa con un enfoque demostrativo, explicativo y propositivo. Como conclusión, los análisis coinciden en lo generalsobre la implementación de la política fiscal implementada en México, la cual ha sido anti cíclica. El incremento en el gasto público no ha contribuido al desarrollo económico, aunque se ha experimentado solo un lento crecimiento económico. Por ser el tema de política fiscal muy extenso y visto desde el aspecto de desarrollo regional solo se hace referencia de investigaciones más recientes que aportan a la investigación de este trabajo, por lo que puede quedar fuera investigaciones realizadas importantes.

\section{MODELO TEÓRICO-CONCEPTUAL SOBRE POLÍTICA FISCAL}

A continuación se presenta el modelo teórico-conceptual sobre la política fiscal que se propone para el análisis. Como ya se comentó, se considera la política fiscal como una herramienta de estabilización macroeconómica, de redistribución del ingreso y de riqueza, utilizada por el Estado en sus tres niveles de gobierno, federal, estatal y local, cuyos componentes principales son el gasto público, los impuestos y la deuda pública, y que se materializan por medio del presupuesto del Estado.

La política fiscal, trata primordialmente de las estrategias que el Estado implementa, no solo para generar recursos, sino también, para administrar de forma eficiente dichos recursos, de allí surge su relación con las finanzas públicas (llamada también política financiera) que incluye los gastos públicos, las deudas públicas y los presupuestos (llamada también política presupuestaria); a su vez, tal política financiera forma parte de la política fiscal enmarcada dentro de la política económica, como podemos ver en la figura 1 siguiente. 
Con respecto a la política financiera, Domínguez (2009), explica secuencia lógica e histórica de las finanzas públicas en tres etapas:

a) La primera consiste en la juridizacionde las finanzas públicas, o sea que la actividad financiera publica ya no puede hacerse más de cualquier manera, no se recauda ni se gasta de cualquier manera, sino que existen criterios jurídicos para determinar ingresos y erogaciones.

b) La segunda etapa consiste en la legalización de la actividad financiera, es el Poder legislativo dentro de la zona de reserva de ley, el que esta exclusivamente habilitado para generar normas, pautas y criterios básicos a los que deben sujetarse los comportamientos erogatorios y recaudatorios.

c) La tercera etapa consiste en la constitucionalizacionde la actividad financiera, esto significa que la actividad de generación jurídica que realice el órgano legislativo deberá hacerse dentro del marco supremo que postule la Constitución.

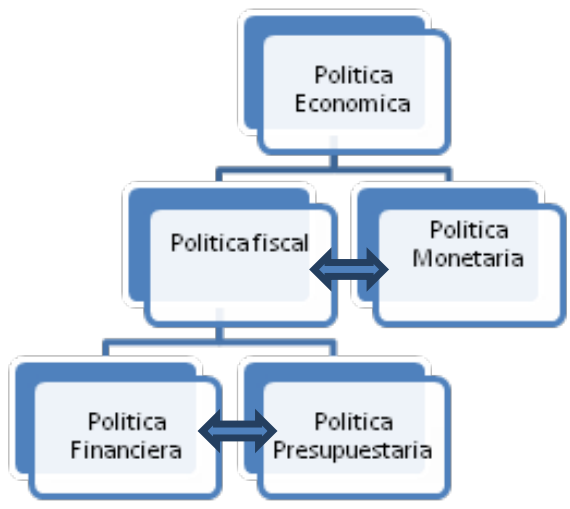

Figura 1: Política fiscal y su aspecto interdisciplinario. Fuente: Elaboración propia (2014). 
El aspecto normativo de política fiscal se vincula con el derecho financiero constitucional, sobre todo por la actividad financiera del Estado estando normada en la constitución existiendo criterios jurídicos para determinar los ingresos y gastos del Estado.La política fiscal no solo debe respetar la constitución, sino que debe contribuir a su realización, siendo un principio básico que debe respetar el estado a la hora de elaborar políticas públicas.

Cabe señalar que, desde el sistema tributario y de su administración, se debe considerar a la política fiscal como un elemento del propio sistema tributario conjuntamente con el derecho tributario, la administración tributaria, y el comportamiento del contribuyente (Toninelli, 2004), ubicándola dentro de la política económica y atribuyéndole las funciones de determinar el monto de los impuestos que se pretende recaudar, sobre qué sectores, de qué manera y con qué tributos. Así como se ha visto que la política fiscal debe enmarcarse en la Constitución, también en el derecho, y en particular el derecho tributario.

Finalmente, de acuerdo a lo anteriormente señalado, se puede señalar que el objetivo principal de la política fiscal es lograr un crecimiento sostenido de la economía por medio del control de la inflación, el desempleo y planificación de la de la producción entre otras variables.

\section{REFERENCIAS}

Angulo López, E. (2011). Política fiscal y estrategia como factor de desarrollo de la mediana empresa comercial sinaloense: Un estudio de Caso. Tesis doctoral, Universidad Autónoma de Sinaloa.

Arrioja Vizcaíno, (2010).Derecho Fiscal. D.F., México: Themis.

Asorey, R. O. (s.f.).Introducción a la política fiscal. Recuperado de App. vlex.com.wdg.biblio.udg.mx:2048/\#WW/search/*/politica+ fiscal/ vid/515952550/graphical_version 
Brue, S. L. y Grant, R. R. (2009). Historia del pensamiento económico (séptima edición). México: CENGAGE Learning.

Caballero Urdiales, E. (2006).Los ingresos tributarios del sector público de México. México: Universidad Autónoma de México, Facultad de economía.

Cárdenas Cutiño, G. A. (2008). El Diccionario de Ciencias Económicas Administrativas (quinta edición). México: CUCEA, Centro Universitario de Ciencias Económico Administrativas.

Centro de emprendedores de la administración pública, (s.f.).Glosario de finanzas públicas locales. Recuperado de www.ccaorg.mx/funcionarios/ emprendegestionpublica/glosario fina.html

Chávez Martín del Campo, J. C., Rodríguez Vargas, R., Fonseca Hernández, F. J. (2010, Julio-Diciembre). Vacas gordas y vacas flacas: La política fiscal y el balance estructural en México, 1990-2009. Estudios Económicos. Fecha de consulta: 9 de marzo de 2015. Recuperado de <http://www.redalyc.org/articulo.oa?id=59716168002 >ISSN 0188-6916

Córdoba Padilla, M. (2009). Finanzas públicas, soporte para el desarrollo del Estado. (segunda edición). Colombia: EOOE Ediciones.

Cortes, F. (2010).Desigualdad económica y poder en México. D.F., México: N.U., CEPAL, Sede Subregional en México.

CPEUM (2015). Leyes federales vigentes, Versión electrónica, Recuperada de http://www.diputados.gob.mx/LeyesBiblio/ref/cpeum.htm

Cruz Núñez, I. (2010). Política fiscal en México bajo el modelo neoliberal (1982-2009) ¿Obstáculo al crecimiento económico? Tesis de maestría estudios sociales, línea economía social, Universidad Autónoma Metropolitana, Unidad Iztapalapa, División de ciencias sociales y humanidades.

Cuestas Caza, J. A. (2013). La política fiscal implementada en Ecuador y su relación con los modelos alternativos de desarrollo 2007-2012. Recuperado de http://repositorio.educacionsuperior.gob.ec/handle/28000/772

Domínguez, A. G. (2009).Constitución económica y derechos económicos, sociales y culturales. Buenos Aires, Argentina, Ad-Hoc.

Erías Rey, A. y Sánchez Santos, J. M. (1998).Política monetaria y política fiscal. Madrid, España: Pirámide, serie economía y gestión internacional. 
Estada Alvarado, M. M. y Gómez Camacho, N. (2000). La política fiscal en México como palanca de crecimiento: Estimulo u obstáculo. Tesis de licenciatura en economía, Universidad Autónoma Metropolitana (UAM).

Galindo Martin (coord.) (2008).El Diccionario de Economía Aplicada: Política económica, economía Mundial y estructura económica. Madrid: Ecobook-Editorial del Economista.

Galindo Martín, M. A. (2007, Marzo-Abril).Gobernanza, política fiscal y crecimiento económico. Nuevas tendencias en política fiscal. N. 835 ICE, Universidad de Castilla-La Mancha.

García Villarejo, A. y Salinas Sánchez, J. (s.f.). Manual de Hacienda Pública, general y de España. España: Tecnos.

Hebe Balbo, E. (2010). El derecho presupuestario a ser feliz. Periódico económico tributario, No. 434, p.8. Recuperado de http://www. eco-finanzas.com/diccionario/D/DESEMPLEO.htm

Inzunza Mejía, P. C. y Sánchez Díaz, A. J. (2013). Disciplina fiscal: límites de la política fiscal para promover el desarrollo en México. Nóesis. Revista de Ciencias Sociales y Humanidades, 22(43) 135-160. Recuperado de http:// www.redalyc.org/articulo.oa?id=85927874006

KeynesMaynard, J. (1936). Teoría general de la ocupación, el interés y el dinero (edición limitada: cuarta edición, corregida y aumentada, 2003). D.F., México: fondo de Cultura Económica.

Manzano, B. y Ruiz, J. (2004). Política fiscal óptima: El estado de la cuestión. Investigaciones Económicas, XXVIII (1) 5-41. Recuperado de http:// www.redalyc.org/articulo.oa?id=17328101

Martínez, D. L., Caamal Cauich, I. y Ávila Dorantes, J. A. (2011, septiembre 10). La política fiscal y el crecimiento económico en México. Análisis del medio rural latinoamericano 137. Recuperado de http://www. bibliocolabora.es.wdg.biblio.udg.mx:2048/ConexionWeb/

Martin-Mayoral, F. y Uribe, C. A. (2010, julio-septiembre). Determinantes económicos e institucionales del esfuerzo fiscal en América Latina. Revista Investigación Económica, vol. LXIX, 273, pp. 85-113.

Merino Peral, M. C y López García, F. J. (2006). Diccionario Enciclopédico de Economía, Finanzas y Empresa. Burgos: Universidad de Burgos, Servicio de Publicaciones. 
Moleres Regalado, E. (2012). Política fiscal, tipo de cambio y tasa natural de crecimiento en México: Evidencia empírica. Tesis de maestría en economía, Universidad Nacional Autónoma de México.

OCDE (2008).Política fiscal y desarrollo en América Latina: en busca del vínculo: Resumen ejecutivo.Perspectivas económicas de América Latina 2009. http://dx.doi.org/ 10.1787/4710360380.

Oszlak, O. (Jan. - Mar., 1982). Reflexiones sobre la formación del Estado y la construcción de la sociedad argentina. Instituto deDesarrollo Económico y social, Vol. 21, No. 84, pp. 531-548, URL: http://www.jstor.org/ stable/3466613

Pliego Quintana, M. (2012). Política fiscal en situaciones recesivas: 1993 2004. Tesis doctoral en economía, Universidad autónoma de México.

Pliego Quintana, M. (2014, May-August). Análisis coyuntural de la política fiscal en México 1993-2004. Economía UNAM, Volume 11, Issue 32, Pág. 59-76, doi: 10.1016/S1665-952X(14)70452-9

Posner, R. (2010).Reseña de "La teoría general del empleo, el interés, y el dinero" de Jonh Maynard Keynes. Revista de Economía Institucional, 12(22)293-305. Recuperada de http://www.redalyc.org/articulo .oa?id=41915003015

Ramírez Cedillo, E. (2006). Por una política fiscal contraciclica. Problemas del Desarrollo. Revista Latinoamericana de Economía, 37(147) 81-107. Recuperado de http://www.redalyc.org/articulo.oa?id=11820100004

Ramírez Cedillo, E. (2009). El abandono de la política fiscal como herramientas de crecimiento. Tesis doctoral de economía, Universidad Autónoma de México.

RetchkimanK, B. (1976). La política fiscal Mexicana. Problemas del Desarrollo, Revista Latinoamericana de Economía, (Vol. 6), No. 24, México. Recuperado de www.revistas.unam.mx/index.php/pde/article/ viem/41364/37615

Rodríguez Arana, Z. A. (2012). Efectos de las políticas fiscal y monetaria en un modelo donde se determinan simultáneamente el ciclo y el crecimiento económico. Análisis Económico, XXVII (66) 97-120. Recuperado de http:// www.redalyc.org/articulo.oa?id=41326845007

Rodríguez Nava, A. y Venegas Martínez, F. (2012, Septiembre-Diciembre). 
Criterios y medidas de política económica en respuesta a la actual crisis económica y financiera. Argumentos, vol. 25, núm. 70, septiembrediciembre, 2012, pp. 39-58, Universidad Autónoma Metropolitana Unidad Xochimilco, Distrito Federal, México.

Samuelson, P. S. y Nordhaus W. D. (s.f.). Macroeconomía. México: McGraw-Hill.

Santaella, J. A. (s.f.). La viabilidad de la política fiscal: 2000-2025. Una agenda para las finanzas públicas en México. Recuperado de ftp://ftp.itam. $\mathrm{mx} /$ pub/academico/inves/CEA/Capitulo2.pdf

Shively, W. P. (1997). Introducción a las ciencias políticas (quinta edición). México: McGraw Hill.

Smith, A. (1958).Investigación sobre la naturaleza y causas de la riqueza de las naciones (decimoctava reimpresión 2012). México: Fondo de Cultura Económica.

Taylor, J. y Weerapana, A. (2011). Principios de Economía (séptima edición). México: CengageLearning

Toninelli, Á. R. (2004). Tratado de tributación: T. II, parte V. Buenos Aires, Argentina: Administración tributaria.

Vargas Téllez, C. O. (2006). Aplicada Incidencia Fiscal y del Gasto sobre la Renta Familiar: Un Enfoque de Microdatos para México 1984-2002. Tesis Doctoral, Universidad Autónoma de Barcelona, Departamento de Economía.

Vázquez López, R. (2006, junio). Los antecedentes del estancamiento económico: la política fiscal en México de 1984-1994. Comercio Exterior, vol. 56, núm. 6.

Artigo recebido em 10/07/2015

Artigo aprovado em 10/08/2015 